\title{
Aspectos operacionais do controle do Triatoma brasiliensis
}

\author{
Operational aspects of \\ Triatoma brasiliensis control
}

Liléia Diotaiuti 1

Osvaldo F. Faria Filho 2

Francisco C. F. Carneiro 2

João Carlos Pinto Dias 1

Herton Helder R. Pires 1

Christopher J. Schofield 3

\footnotetext{
1 Centro de Pesquisas René Rachou, Fundação Oswaldo Cruz. Av. Augusto de Lima 1715, Belo Horizonte, $M G$ 30190-002, Brasil. diotaiut@cpqrr.fiocruz.br 2 Fundação Nacional de Saúde. Av. Santos Dumont 1856, Fortaleza, CE 60150-160, Brasil.

3 London School of Hygiene and Tropical Medicine. Keppel St., London WCIE 7HT, England.
}

\begin{abstract}
Vector control strategies against indigenous species is not easy, due to their capacity to reinvade treated premises from sylvatic ecotopes. Between August 1996 and December 1997 we conducted a study on reinfestation of houses after spraying in a county in the State of Ceará. Of 277 houses examined, 113 (40.8\%) were infested (21.7\% intradomiciliary and 35.4\% peridomiciliary). Of the 433 Triatominae collected, 207 were Triatoma brasiliensis (49\% of which intradomiciliary, with a mean of 1.8 insects/house) and 226 were Triatoma pseudomaculata (97\% peridomiciliary). The age structure of the two indicated a univoltine development cycle for $\mathrm{T}$. brasiliensis and two cycles per year for T. pseudomaculata. Four months after spraying with deltamethrin SC $25 \mathrm{mg}$ ia/m², 9.7\% of the houses were still positive, mainly with peridomestic infestations. Intradomiciliary wall bioassays showed persistence of the insecticide up to 9 months after spraying. Considering the high potential for recolonization of treated premises from sylvatic foci, we propose an operational strategy combining traditional evaluations and communitybased surveillance with increased selective interventions and community education.
\end{abstract}

Key words Chagas Disease; Triatominae; Vector Control; Insect Vectors; Prevention and Control

Resumo O controle de triatomíneos é dificultado pela capacidade de reinvasão das casas por exemplares silvestres. Entre agosto/96 e dezembro/97 realizou-se, no Ceará, um estudo a respeito da reinfestação das casas após borrifação. Das 277 Unidades Domiciliares - UD - pesquisadas, 40,8\% estavam infestadas (21,7\% dos intradomicílios e 35,4\% dos peridomicílios). Dos 433 triatomíneos capturados, 207 eram Triatoma brasiliensis (48,8\% no intradomicílio, média de 1,8 insetos/casa) e 226 Triatoma pseudomaculata (97,3\% no peridomicílio). Ocorre um único ciclo anual do T. brasiliensis, e dois ciclos anuais do T. pseudomaculata. Quatro meses após a borrifação, 9,7\% das unidades domiciliares permaneciam positivas, principalmente no peridomicílio; 10,3\% das UD foram positivas em todas as avaliações. O teste de suscetibilidade biológica à deltametrina revelou a persistência do inseticida no intradomicílio até nove meses após a borrifação. A prevalência global da infecção humana foi de 5,7\%, tendo sido positivas cinco crianças menores de dez anos. Considerando-se a alta pressão de recolonização a partir de exemplares silvestres, propõe-se, como metodologia de controle, um sistema misto da avaliação tradicional e a vigilância epidemiológica.

Palavras-chave Doença de Chagas; Triatominae; Controle de Vetores; Insetos Vetores; Prevenção e Controle 


\section{Introdução}

A metodologia de controle vetorial da doença de Chagas no Brasil prioriza a borrifação das unidades domiciliares infestadas. Até recentemente, esta borrifação não distinguia as diferentes espécies de triatomíneos, sendo, em um primeiro momento, seletiva por localidade positiva e, a seguir, por unidade domiciliar (UD) positiva. Desta forma obteve-se a eliminação do Triatoma infestans (Klug, 1834) de amplas áreas do território nacional, bem como a redução da infestação e da densidade intradomiciliar das demais espécies igualmente responsáveis pela transmissão humana do Trypanosoma cruzi. Como tentativa de adequação da metodologia às características biológicas das espécies autóctones de triatomíneos com capacidade de recolonização do ambiente artificial após a borrifação a partir de exemplares provenientes do ambiente silvestre, Silveira \& Rezende (1994) propuseram alteração na periodicidade e amplitude da borrifação.

Em função da baixa sensibilidade da técnica de captura manual, da mesma forma que pela alta capacidade de reinfestação do Triatoma brasiliensis (Neiva, 1911), o uso do inseticida contra esta espécie passou a incluir todas as unidades domiciliares, inclusive as negativas, das localidades onde alguma casa tenha sido positiva. Esta metodologia foi recentemente implantada na rotina do Programa de Controle da Doença de Chagas (PCDCh) e, apesar de seus efeitos não terem sido avaliados de modo adequado, já se sabe que é trabalhosa e onerosa. Na verdade, o que se pergunta é se tratamento tão amplo realmente é necessário, uma vez que a metodologia tradicional foi capaz de interromper a transmissão da doença de Chagas em diferentes municípios do Ceará, estado onde predomina o T. brasiliensis. Silveira (1995) destaca a carência de informações relativas à dinâmica populacional da espécie que permitam definir a melhor forma de intervenção.

Em 1996 deu-se início, no estado do Ceará - com o apoio da Fundação Nacional de Saúde (FUNASA) - a uma série de estudos que pudessem responder questões específicas do controle do T. brasiliensis, tais como o potencial de recolonização das casas após a borrifação e o potencial de transmissão vetorial atual nas condições de infestação hodiernas. O presente artigo sumaria os resultados iniciais deste trabalho, como abertura à revisão da corrente metodologia, tendo em vista a formulação de propostas imediatas.

\section{Material e métodos}

O estudo do impacto da borrifação sobre a população de T. brasiliensis realizou-se no Município de Independência (Ceará), situado na região oeste, bastante representativo da situação do T. brasiliensis no Ceará e no Nordeste Brasileiro (Alencar, 1987). Em agosto-setembro/96 foram pesquisadas 277 unidades domiciliares (UD). Todas as UD da área foram borrifadas com deltametrina ( $25 \mathrm{mg} \mathrm{ia} / \mathrm{m}^{2}$ ), que é a dose padrão do PCDCh (Dias, 1987). Novas pesquisas (contagem dos triatomíneos no local, sem captura) foram realizadas em intervalos de cerca de quatro meses, ou seja, em janeiro-fevereiro/97, junho-julho/97 e novembro-dezembro/97. Na avaliação efetuada em novembro e dezembro/96 foram distribuídos sacos plásticos para que os moradores das casas ali depositassem os insetos adultos por eles flagrados em vôo de ingresso na casa. Estes sacos plásticos foram recolhidos em janeiro/97.

A prevalência da doença de Chagas humana no município foi estimada na população geral mediante inquérito soroepidemiológico, coleta de sangue em papel de filtro e processamento por imunofluorescência indireta e hemaglutinação, utilizando-se o ponto de corte de 1:40.

Em Novo Oriente, município vizinho, segue sendo avaliada a suscetibilidade biológica de $T$. brasiliensis procedentes de insetário à deltametrina na dose habitual de $25 \mathrm{mg} \mathrm{ia} / \mathrm{m}^{2}$, e com $12,5 \mathrm{mg}$ ia $/ \mathrm{m}^{2}$. Vinte exemplares de quinto estádio, de tamanho padronizado e alimentados em galinhas, foram expostos às paredes do intradomicílio no dia da borrifação, tal como decorridos três, seis e nove meses. Os insetos foram colocados em contato com as paredes por 72 horas, quando se contabilizou o número de mortos, intoxicados e normais. A cada avaliação, um grupo controle foi exposto em paralelo a paredes não borrifadas. Serão aqui apresentados os resultados obtidos até nove meses após a borrifação das paredes.

\section{Resultados}

Dentre as 277 UD pesquisadas em Independência, 113 mostraram-se infestadas $(40,8 \%)$, encontrando-se $21,7 \%$ dos intradomicílios e $35,4 \%$ dos peridomicílios positivos. Foi capturado um total de 433 triatomíneos, distinguindo-se $47,8 \%$ T. brasiliensis $(48,8 \%$ deles no intradomicílio) e $52,2 \%$ Triatoma pseudomaculata (Correa \& Espinola, 1964) $(97,3 \%$ no peridomicílio), o que representou densidade triato- 
mínica intradomiciliar de 1,8 insetos/casa. O T. brasiliensis foi encontrado principalmente nas salas $(53,2 \%)$, nos quartos $(24,2 \%)$, nas cozinhas $(16,1 \%)$ e em outros lugares não especificados (6,5\%). Apenas seis exemplares de T. pseudomaculata (três ninfas e três adultos) foram capturados em diferentes cômodos do intradomicílio (Figura 1).

Nas avaliações realizadas após a borrifação, a estrutura etária de T. brasiliensis e de T. pseudomaculata capturados revela a ocorrência de ciclo anual único da primeira espécie, com predominância dos adultos nos meses de junho e julho, assim como, provavelmente, dois ciclos anuais para o T. pseudomaculata (Figura 2). Um ano depois, apesar de notar-se diminuição no número de $T$. brasiliensis intradomiciliares, a quantidade de exemplares recolhidos em toda a unidade domiciliar foi estatisticamente superior ao da captura antes da borrifação. Para o mesmo período, o número de T. pseudomaculata capturados foi semelhante (Figura 3 ).

Os ecótopos mais freqüentemente infestados corresponderam aos poleiros de galinhas (33\%), galinheiros $(23,8 \%)$, currais de cabra ou ovelha $(13,8 \%)$, montes de telha $(10,8 \%)$, chiqueiros $(6,9 \%)$, estábulo de gado $(3,1 \%)$, entre outros de menor importância.

Na primeira avaliação feita após a borrifação - ou seja, quatro meses depois $-9,7 \%$ das unidades domiciliares permaneciam positivas, em particular, no peridomicílio; $10,3 \%$ das UDs foram positivas em todas as avaliações - ou seja, aos 4, 8 e 12 meses após a borrifação (Figura 4). O teste de suscetibilidade biológica dos triatomíneos à deltametrina foi dose dependente, revelando persistência do inseticida na maior dose até nove meses após a borrifação (100\% de mortalidade dos insetos expostos ao contato), e nenhuma ação inseticida na menor dose a partir do sexto mês (Figura 5).

Entre o período de novembro/96 a janeiro/ 97 foi relatado o ingresso de adultos de triatomíneos em 34 UD (Tabela 1).

$\mathrm{O}$ inquérito soroepidemiológico revelou prevalência global de 5,7\% de infecção. A faixa etária de 0-10 anos incluiu 657 crianças, das quais cinco positivas $(0,8 \%)$ (Tabela 2$)$, aumentando progressivamente nas demais faixas até $12,6 \%$ nas pessoas acima de 50 anos.

\section{Discussão}

O controle do $T$. brasiliensis, sem dúvida, é desafiador, e exige medidas mais extensas do que a tradicional metodologia do Programa de Controle da Doença de Chagas. A pressão de reco-
Figura 1

Distribuição de amostra de triatomíneos intradomiciliares capturados em Independência, Ceará.

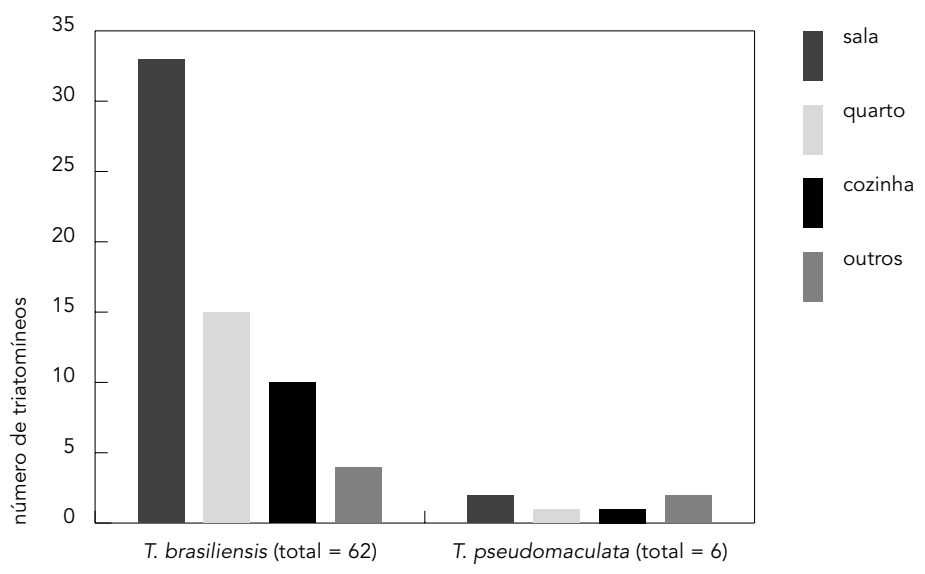

no casas $=60$

Tabela 1

Triatomíneos que invadiram as casas voando, capturados pelos moradores no período de novembro/97 a janeiro/98. Município de Independência, Ceará, área rural.

\begin{tabular}{lccc}
\hline & T. brasiliensis & P. lutzi & T. pseudomaculata \\
\hline Machos & 14 & 17 & 6 \\
Fêmeas & 44 & 1 & 3 \\
Total & 58 & 18 & 9 \\
\hline
\end{tabular}

$\mathrm{UD}=34$

Tabela 2

Inquérito soroepidemiológico em amostras da população rural do Município de Independência, Ceará, Brasil, 1997.

\begin{tabular}{lc}
\hline Faixa etária $(\mathbf{n})$ & Prevalência \\
\hline $0-10$ anos (657) & $0,8 \%$ \\
$11-15(302)$ & $2,3 \%$ \\
$16-20(245)$ & $4,9 \%$ \\
$21-30$ anos (337) & $5,6 \%$ \\
$31-40$ anos (242) & $7,0 \%$ \\
$41-50$ anos (207) & $10,6 \%$ \\
$>50$ anos (460) & $12,6 \%$ \\
Total (2.450) & $5,7 \%$ \\
\hline
\end{tabular}


Figura 2

Percentual relativo de captura de adultos e ninfas de T. brasiliensis e T. pseudomaculata no Município de Independência, Ceará, Brasil.
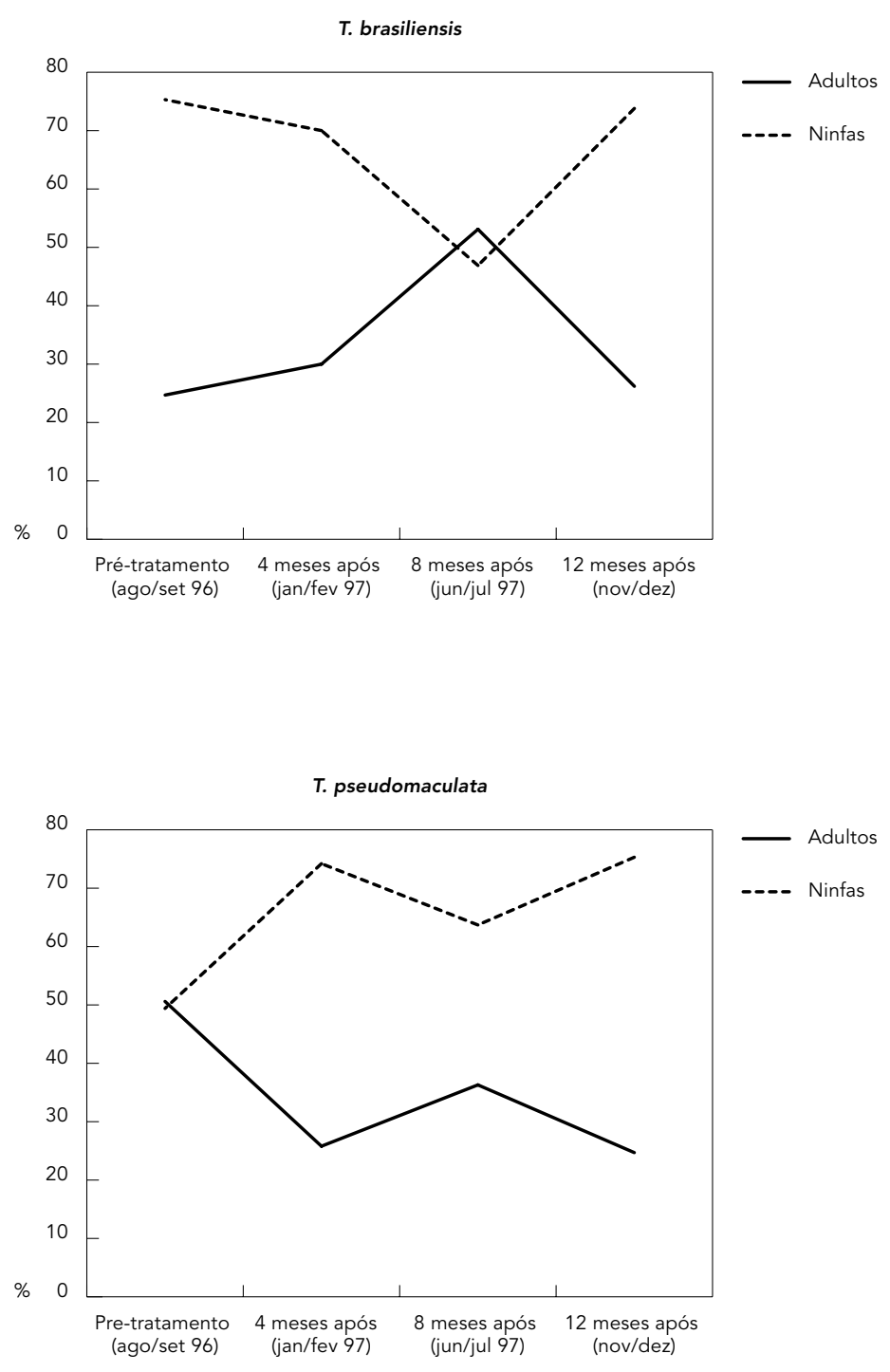

lonização das casas a partir dos focos silvestres é grande, como se pode observar pelo número de exemplares adultos que ingressaram nas casas voando, comprovando, na região, os relatos históricos de Alencar (1987). O potencial de reinfestação pelo T. brasiliensis aqui demonstrado é realmente elevado, uma vez que as colônias se reconstituíram no período de um ano apesar da remoção dos insetos a cada avaliação. Fora isso, o fato de serem encontradas 9,7\% unidades positivas quatro meses após a borrifação, com colônias principalmente peridomiciliares constituídas por triatomíneos de todos os estádios, evidencia que muitos insetos não são atingidos pelo inseticida e sobrevivem, reconstituindo as colônias originais no prazo máximo de um ano. Este fato não é difícil de ser compreendido, uma vez que tanto as casas como os anexos podem apresentar inúmeros esconderijos inacessíveis à borrifação, mesmo que o inseticida apresente ação residual no intradomicílio.

A hipótese de resistência ao inseticida pelo T. brasiliensis na região pode ser descartada, em virtude de terem sido utilizados, para os testes de suscetibilidade biológica aqui apresentados, triatomíneos descendentes de exemplares capturados nesta área que se revelaram suscetíveis à deltametrina. Portanto, o mais provável é que a recolonização seja decorrente de triatomíneos de procedência silvestre e dos originários do peridomicílio.

Por sua vez, a elevada infestação das salas pode encontrar justificativa em seu uso como dormitório, pois os moradores estendem as redes, à noite, nesse local, pernoitando aí também animais como cães, gatos e galinhas.

Interessante observar que, apesar da presença de colônias intradomiciliares de T. brasiliensis, a prevalência da infecção humana $(5,7 \%)$ não é alta. Mereceria investigação mais acurada o hábito regional de dormir em redes, o que certamente diminui o contato com os triatomíneos e pode evitar a infecção. O encontro de triatomíneos nas redes não é raro, mas em número bem menor do que o que se pode encontrar nas camas - tidas como ambientes mais estáveis -, uma vez que as redes são recolhidas diariamente ao amanhecer. Ainda assim, a transmissão está ocorrendo na área investigada $(0,8 \%$ de prevalência na faixa etária abaixo de dez anos), o que, por si só, constitui fato extremamente grave, exigindo especial atenção e definindo a necessidade de tratamento específico (Dias, 1997).

Neste trabalho não tivemos oportunidade de avaliar os efeitos da borrifação das casas negativas vizinhas às positivas. Esta medida teria 
sentido no caso de considerar-se que a técnica de captura na rotina dos trabalhos é limitada, além de que alguma casa dada como negativa possa ser, na verdade, positiva. Entretanto, julgar que uma UD venha a servir como foco básico de infestação para as outras é contra-senso, uma vez que a investigação da infestação de ecótopos silvestres revela um panorama em que predominam as pedras, sob as quais podem ser encontradas inúmeras colônias de triatomíneos associados a roedores, com grande número destes infectados pelo T. cruzi. Portanto, o grande potencial de reinfestação é representado pelo ambiente natural da caatinga, no qual a inserção do ambiente artificial tem pouca importância. A instalação da vigilância epidemiológica tal qual proposta pela Fundação Nacional de Saúde - ou seja, municipalizada e em substituição às avaliações verticais poderá redundar em problemas operacionais. Face às altíssimas taxas de dispersão, espera-se a ocorrência de expressivo número de notificações concomitantes de focos por parte da população, o que trará dificuldades operacionais em seu atendimento, demandando um poder de resposta que a maioria dos municípios ainda não tem (Dias, 1998). Claro está que, desde que bem realizadas, as tradicionais avaliações exercem impacto significativo sobre a população de T. brasiliensis, e podem interromper a transmissão da doença de Chagas (Ledebour et al., 1995; Silveira, 1995). Em decorrência do exposto, é recomendável que se realize análise minuciosa acerca da forma como tem sido realizado o Programa de Controle da Doença de Chagas no Nordeste, antes de se criarem falsos conceitos no que diz respeito ao potencial biológico da espécie ou a mitos de resistência a inseticida ou, ainda, quanto a uma provável maior transitoriedade dos piretróides no Nordeste, em razão das temperaturas altas e da intensa luminosidade. Algumas inovações metodológicas poderão ser interessantes, tais como um sistema misto da avaliação tradicional e vigilância. No Triângulo Mineiro, área colonizada pelo Triatoma sordida (Stal, 1859) e no qual a infestação persistia e se reconstituía rapidamente após as borrifações anuais, borrifações semestrais foram capazes de controlá-la por período superior a um ano (Dias, 1995). Fato semelhante foi observado por Ledebour et al. (1995).

No caso do T. brasiliensis, poder-se-ia também pensar no aumento da pressão inseticida, acompanhada da vigilância epidemiológica, de maneira a ter-se controle efetivo e rápido sobre os novos focos, abrindo espaço igualmente para atividades educativas, que contemplem a melhoria física e do manejo dos ecótopos peri-
Número de exemplares de triatomíneos capturados no Município de Independência, Ceará, Brasil (1996/1997).
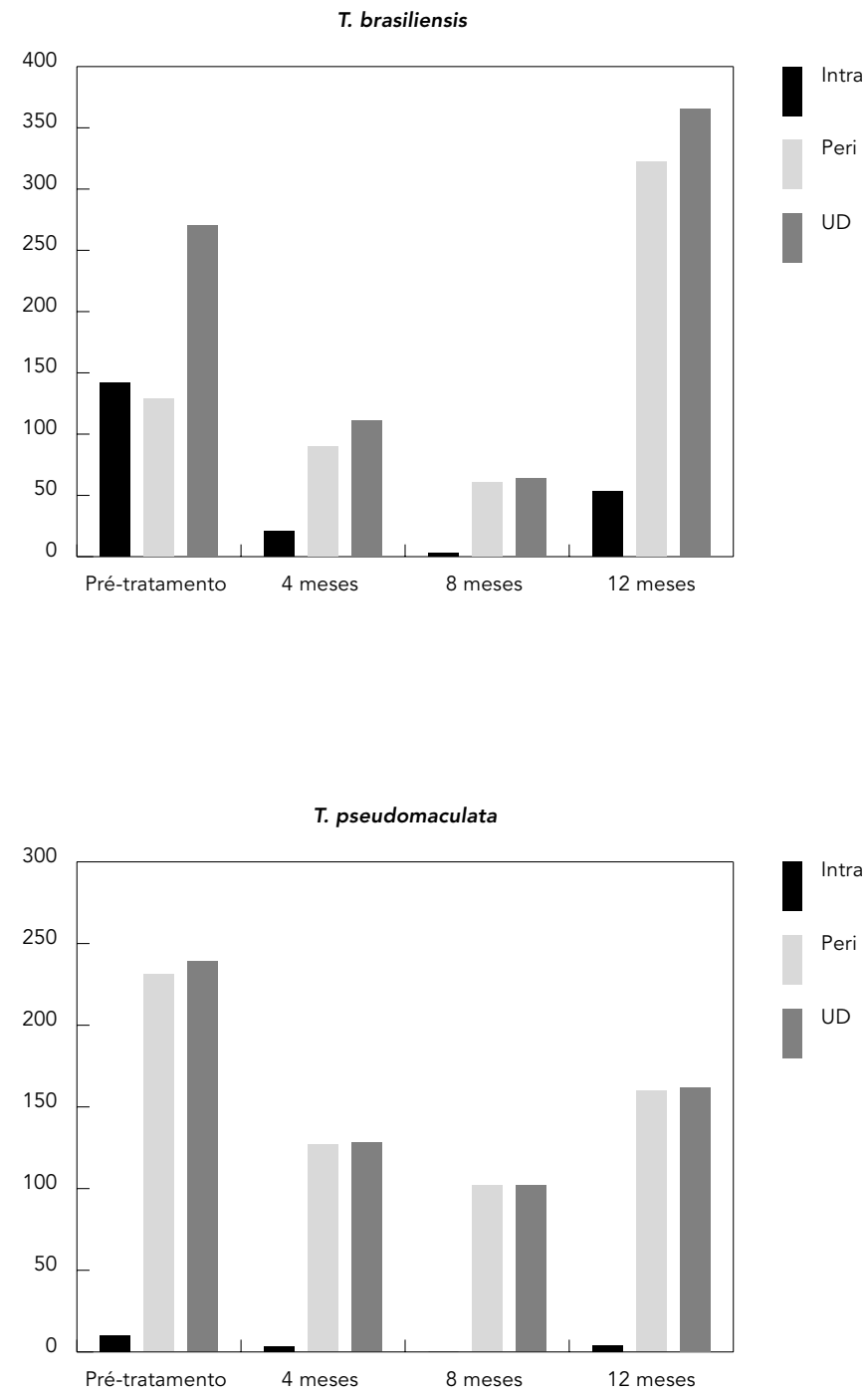
Figura 4

Acompanhamento da infestação de 155 Unidades Domiciliares positivas no pré-tratamento com deltametrina SC $25 \mathrm{mg}$ ia/m² até 12 meses após a borrifação, no Município de Independência, Ceará, Brasil.

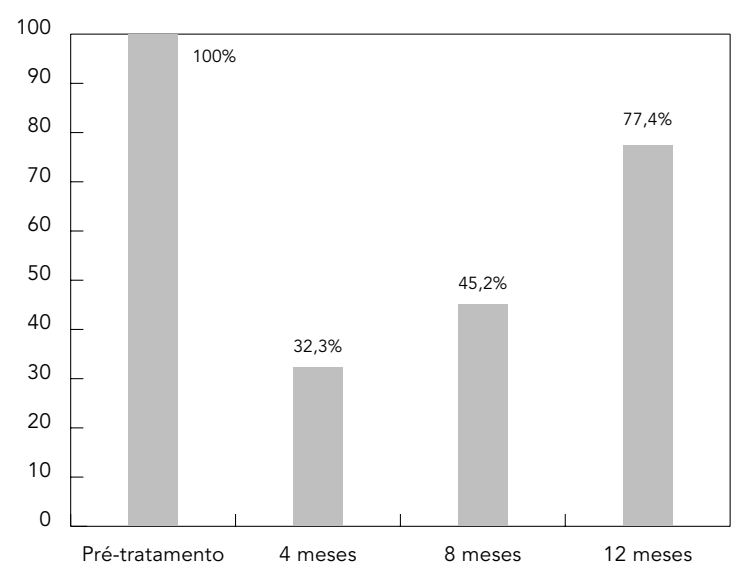

Positivas no:

pré-tratamento +4 meses: 15 UD $(9,7 \%)$

pré +4 meses +8 meses: 8 UD $(5,2 \%)$

pré +4 meses +8 meses +12 meses: 16 UD $(10,3 \%)$ domiciliares, em especial, aqueles que melhor e mais comumente albergam os triatomíneos em questão, como os poleiros e galinheiros na presente observação (56,8\% dos focos).

Dentro da realidade do Programa de Controle da Doença de Chagas no Brasil, os estados da Região Nordeste apresentam flagrante descompasso com os demais em relação ao sucesso na eliminação de colônias intradomiciliares de triatomíneos. Entretanto, a análise dos relatórios de atividade da Fundação Nacional de Saúde expressa que, nesta região, o trabalho não alcançou os índices de continuidade e contigüidade que definiram este programa em outras áreas, nas quais se logrou a interrupção da transmissão vetorial e a dramática redução na distribuição do Triatoma infestans, que correspondia ao principal vetor nestas áreas (Dias \& Schofield, 1999). Por conseguinte, ao iniciarmos uma avaliação acerca de aspectos operacionais no controle do T. brasiliensis, é fundamental a extrapolação para um contexto que transcende os fenômenos biológicos, podendo ser bastante limitado por aspectos políticos e de distribuição dos recursos na área de saúde. Trabalhos anteriores revelaram a competência dos técnicos locais e a eficiência e adequação da metodologia de rotina deste programa no Estado do Ceará, deste que obedecidas as suas regras básicas e, em especial, onde o trabalho obteve a cobertura temporal e espacial necessárias (Silveira, 1995). Entretanto, a falta de informações a respeito da biologia das espécies nordestinas abre espaço a perguntas fundamentais, que poderiam melhor subsidiar as atividades de controle. Por exemplo, na última tentativa de normatização da borrifação para sua adequação às solicitações de cada espécie, as áreas infestadas pelo T. brasiliensis deveriam ser borrifadas integralmente, ou seja, todas as unidades domiciliares da localidade positiva deveriam ser borrifadas, inclusive as negativas. Naturalmente, tal norma envolve aumento expressivo nas atividades de campo e, em conseqüência disso, de custo. Se com a orientação anterior (borrifação apenas das UD positivas) já não se conseguia cobrir integralmente as áreas infestadas, com a nova norma este problema aumentou ainda mais, de forma que é praticamente impossível fechar as atividades de captura e borrifação de um município no mesmo ano contando apenas com os recursos e a carência de mão de obra atuais.

Efetivamente, o futuro do controle dos vetores da doença de Chagas é representado pela vigilância epidemiológica, pautado não só pelos seus índices entomológicos, mas, principalmente, pela própria orientação do SUS, que di- 
reciona as atividades de saúde para o nível municipal (U. Pedrosa, comunicação pessoal). É importante, entretanto, considerar a necessidade de manutenção - provavelmente, a nível estadual - de estrutura sanitária capaz de atender aos pressupostos básicos para o controle de determinadas endemias, no caso, a doença de Chagas, com planejamento que vislumbre a continuidade temporal e contigüidade espacial capazes de garantir o sucesso do programa (Dias, 1998). Se assim for, é provável que um esquema de borrifação com alta pressão inseticida inicial, vigilância epidemiológica paralela e ciclos de avaliação vertical bi ou tri-anual venha a adequar-se às necessidades epidemiológicas e políticas para o controle do T. brasiliensis.

Como necessidade sentida, linhas complementares de investigação já estão em andamento no tocante à determinação do papel dos focos silvestres no processo de reinfestação das

\section{Agradecimentos}

À equipe de campo da Fundação Nacional de Saúde, Subdistrito de Crateús, Ceará; em especial, aos Srs. José Evanildo Fernandes de Souza e Francisco Diassis Vieira. unidades domiciliares, através da caracterização química e morfológica das população de insetos e também do T. cruzi circulante na região. Igualmente faz-se mister o aprimoramento de instrumentos e estratégias de detecção precoce de triatomíneos na vigilância - em especial, no peridomicílio (Dias, 1998). Concomitante a isso, é fundamental uma linha de pesquisas operacionais que contemple as possibilidades e dificuldades do trabalho municipalizado, assim como tentativas de sua racionalização, em termos de sustentabilidade, continuidade, efetividade e relação custo-benefício.

É também prudente e necessário avaliar as condições atuais de trabalho da FUNASA na área, analisando-se, em profundidade, riscos, perspectivas e eventuais alternativas quanto à propalada extinção desta entidade, atualmente a única responsável pelo controle dos triatomíneos na região.

\section{Referências}

ALENCAR, J. E., 1987. História Natural da Doença de Chagas no Estado do Ceará. Fortaleza: Imprensa Universitária, Universidade Federal do Ceará.

DIAS, J. C. P. \& COURA, J. R., 1997. Epidemiologia. In: Clínica e Terapêutica da Doença de Chagas. Uma Abordagem para o Clínico Geral (J. C. P. Dias \& J. R. Coura, org.), pp. 33-66, Rio de Janeiro: Editora Fiocruz.

DIAS, J. C. P., 1987. Control of Chagas disease in Brazil. Parasitology Today, 3:336-341.

DIAS, J. C. P., 1998. Problemas e possibilidades de participação comunitária no controle das grandes endemias no Brasil. Cadernos de Saúde Pública, 14:19-37.

LEDEBOUR, C. C.; MOREIRA, E. F.; FERREIRA, W. L. B. \& DIAS, J. C. P., 1995. Diferentes esquemas e formulações de lambdacialotrina (Icon ${ }^{\circledR}$ - OMS 3210) no controle de Triatoma brasiliensis em Passira, PE, Brasil (1993/1994). Revista da Sociedade Brasileira de Medicina Tropical, 28 (Sup. 1): 39.

SCHOFIELD, C. J. \& DIAS, J. C. P., 1999. The Southern Cone Iniciative against Chagas disease. Advances in Parasitology, 42:1-27.

SILVEIRA, A. C. \& REZENDE, D. F., 1994. Epidemiologia e controle da transmissão vetorial da doença de Chagas no Brasil. Revista da Sociedade Brasileira de Medicina Tropical, 27:11-22.

SILVEIRA, A. C., 1995. O controle vetorial da doença de Chagas no Brasil - Resultados e perspectivas. In: International Workshop on Population Genetics and Control of Triatominae, Abstracts, pp. 8994, México, D.F.: Instituto Nacional de Diagnóstico y Referencia Epidemiológicos. 